\title{
RESEARCH
}

Open Access

\section{4-Phenyl-1,3-thiazole-2-amines as scaffolds for new antileishmanial agents}

\author{
Carina Agostinho Rodrigues', Paloma Freire dos Santos ${ }^{1}$, Marcela Oliveira Legramanti da Costa', \\ Thais Fernanda Amorim Pavani ${ }^{1}$, Patrícia Xander ${ }^{2}$, Mariana Marques Geraldo ${ }^{2}$, Ana Mengarda ${ }^{3}$, \\ Josué de Moraes ${ }^{3}$ and Daniela Gonçales Galasse Rando ${ }^{1^{*}}$ (D)
}

\begin{abstract}
Background: There is still a need for new alternatives in pharmacological therapy for neglected diseases, as the drugs available show high toxicity and parenteral administration. That is the case for the treatment of leishmaniasis, particularly to the cutaneous clinical form of the disease. In this study, we present the synthesis and biological screening of eight 4-phenyl-1,3-thiazol-2-amines assayed against Leishmania amazonensis. Herein we propose that these compounds are good starting points for the search of new antileishmanial drugs by demonstrating some of the structural aspects which could interfere with the observed activity, as well as suggesting potential macromolecular targets.
\end{abstract}

Methods: The compounds were easily synthesized by the methodology of Hantzsch and Weber, had their purities determined by Gas Chromatography-Mass spectrometry and assayed against the promastigote forms of Leishmania amazonensis as well as against two white cell lines (L929 and THP-1) and the monkey's kidney Vero cells. PrestoBlue ${ }^{\oplus}$ and MTT viability assays were the methodologies applied to measure the antileishmanial and cytotoxic activities, respectively. A molecular modeling target fishing study was performed aiming to propose potential macromolecular targets which could explain the observed biological behavior.

Results: Four out of the eight compounds tested exhibited important anti-promastigote activity associated with good selectivity indexes when considering Vero cells. For the most promising compound, compound $6, I_{50}$ against promastigotes was 20.78 while SI was 5.69. Compounds 3 (IC $50: 46.63 \mu \mathrm{M} ; \mathrm{SI}: 26.11)$ and 4 (IC 50 : $53.12 \mu \mathrm{M}$; SI: 4.80) also presented important biological behavior. A target fishing study suggested that S-methyl-5-thioadenosine phosphorylase is a potential target to these compounds, which could be explored to enhance activity and decrease the potential toxic side effects.

Conclusions: This study shows that 4-phenyl-1,3-thiazol-2-amines could be good scaffolds to the development of new antileishmanial agents. The S-methyl-5-thioadenosine phosphorylase could be one of the macromolecular targets involved in the action.

Keywords: 2-aminothiazoles, Antikinetoplastids, Antileishmanial, Cutaneous, Target fishing

\footnotetext{
* Correspondence: dgrando@unifesp.br

${ }^{1}$ Chemical and Pharmaceutical Research Group, Department of

Pharmaceutical Sciences, Institute of Environmental, Chemical and

Pharmaceutical Sciences, Federal University of São Paulo (UNIFESP), Rua São

Nicolau, 210, 20 andar, Diadema, SP 09913-030, Brazil

Full list of author information is available at the end of the article
}

(c) The Author(s). 2018 Open Access This article is distributed under the terms of the Creative Commons Attribution 4.0 International License (http://creativecommons.org/licenses/by/4.0/), which permits unrestricted use, distribution, and reproduction in any medium, provided you give appropriate credit to the original author(s) and the source, provide a link to the Creative Commons license, and indicate if changes were made. The Creative Commons Public Domain Dedication waiver (http://creativecommons.org/publicdomain/zero/1.0/) applies to the data made available in this article, unless otherwise stated. 


\section{Background}

Neglected diseases affect thousands of people, predominantly in developing countries [1]. The available pharmacological therapies, however, are far from satisfactory, since the existing drugs exhibit a moderate efficacy associated with toxicity. This is the case of leishmaniasis, a tropical endemic disease caused by protozoa of the genus Leishmania [2]. The most common leishmaniasis treatments frequently require parenteral administration and chronic therapeutic schemes, which result in high costs and low accessibility to the farthest endemic regions [3]. For these reasons, the need for new alternatives to treat neglected diseases remains urgent.

The 2-aminothiazole scaffold is present in the structure of antiviral [4], antifungal [5], antimicrobial [6], anti-cancerous [7], and anti-inflammatory compounds [8]. Recent papers have reported different 2-aminothiazole derivatives as promising agents against kinetoplastids such as Trypanosoma cruzi, Trypanosoma brucei, Leishmania donovani, and Leishmania infantum [9-11].

Kaiser et al. screened a set of 400 drug-like compounds from the chemical library known as "Malaria Box", made available by the Medicines for Malaria Venture initiative, for potential repurposing of these compounds as antikinetoplastids. Their findings showed that 2-aminothiazole derivatives might be potential hits to be explored against trypanosomatids, specifically against Trypanosomes [9].

More recently, Papadopoulou et al. synthesized a small series of 5-nitro-2-aminothiazoles derivatives which presented better antitrypanosomal activity than the standard drug benznidazole. Antileishmanial activity was also observed in some of the derivatives; however, toxicity against L6 cells was also verified [10].

The 2-aminothiazole ring has gained increasing attention as an antiprotozoal hit. Nevertheless, the 4-phenyl-1,3-thiazole-2-amine scaffold alone has never been explored by its biological potential, mainly regarding the antileishmanial activity.

In this paper, we report the synthesis and biological screening of eight 4-phenyl-1,3-thiazol-2-amines assayed against L. amazonensis, a species of leishmania from the L. mexicana complex, which is responsible for the cutaneous form of the disease. The compounds were also explored as to assess their cytotoxicity, selectivity index, and potential macromolecular targets.

The main objective of these studies was to understand whether 4-phenyl-1,3-thiazol-2-amines could be good starting points in the search for new antileishmanial drugs, as well as to assess the chemical aspects that could interfere with the activity. Potential macromolecular targets, which could explain the observed actions, were also investigated by target fishing approach.

\section{Methods}

Eight different aromatic ketones were applied in accordance with their 4-phenyl ring substituent, considering their effect on the lipophilicity of the system (Table 1).

\section{Synthesis}

The 4-phenyl-2-aminothiazoles were synthesized according to the methodology of Hantzsch and Weber [12], from their respective arylketones and thiourea (1:2), in the presence of iodine ( 4 equivalents), by heating the mixture for $4 \mathrm{~h}$. At the end of the reaction, hot water was added to the raw mixture, which was cooled to room temperature and washed with three portions of ethyl ether $(\sim 10 \mathrm{~mL}$ each) to remove the residual iodine. The water phase was neutralized with a saturated solution of ammonium hydroxide and washed with distilled water to yield a solid isolated by filtration under reduced pressure. The pure 4-phenyl-1,3-thiazol-2-amines were obtained by crystallization from Ethanol:water (4:1) solution [13].

\section{Antileishmanial assay}

All compounds were assayed against promastigotes of Leishmania amazonensis (MHOM/BR/1973/M2269). Promastigotes were grown in 199 media (Sigma, USA) supplemented with $10 \%(\mathrm{v} / \mathrm{v})$ of fetal bovine serum, $1 \%$ penicillin $(10,000 \mathrm{UI} / \mathrm{mL}) /$ streptomycin $(10.0 \mathrm{mg} / \mathrm{mL})$ (Sigma, USA). The parasites were collected at log phase, they were counted at the Neubauer chamber, their concentration was adjusted to $1 \times 10^{6}$ cell $/ \mathrm{mL}$, and their cells were seeded at a 96-wells plate. The 4-phenyl-2-aminothiazoles were added to the culture, considering concentrations ranging from 3.2 to $142 \mu \mathrm{M}$. Compounds were diluted in Dimethyl sulfoxide (DMSO) (25 mg/mL) and diluted in 199 media to generate a $1 \%$ DMSO starting solution of each test compound. Positive control with no compounds and DMSO control were considered at the assay. All plates were incubated at $25{ }^{\circ} \mathrm{C}$ for $48 \mathrm{~h}$, and PrestoBlue ${ }^{\bullet}$ vital dye was added to the wells.

\section{Table 1 The 4-Phenyl-1,3-thiazol-4-amines series}

\begin{tabular}{lllll}
\hline Compound & $\mathrm{R}$ & $\mathrm{CLogP}$ & $\mathrm{MR}^{\mathrm{b}}$ & Yield \% \\
\hline 01 & $\mathrm{H}$ & 2.61 & 50.20 & 61 \\
02 & $4-\mathrm{CH}_{3}$ & 3.07 & 55.25 & 72 \\
03 & $4-\mathrm{CH}_{2} \mathrm{CH}_{3}$ & 3.47 & 59.85 & 59 \\
04 & $3,4-\mathrm{Cl}$ & 3.64 & 59.81 & 53 \\
05 & $4-\mathrm{CF}_{3}$ & 3.49 & 56.18 & 18 \\
06 & $4-\mathrm{C}\left(\mathrm{CH}_{3}\right)_{3}$ & 4.23 & 68.87 & 27 \\
07 & $4-\mathrm{OH}$ & 2.32 & 52.19 & 47 \\
08 & $4-\mathrm{Cl}$ & 3.12 & 55.01 & 67 \\
\hline
\end{tabular}

(a) Calculated n-Octanol/Water Partition Coefficient; (b) Molar Refractivity 
Viability was accessed with PrestoBlue ${ }^{\bullet}$ (Invitrogen, ThermoFisher) according to the manufacturer's instructions. The reagent was added in each well $(10 \mu \mathrm{L})$ and plates incubated for $2 \mathrm{~h}$. Then, plates were read on a microplate reader (PowerWave HT, Biotek) with excitation/emission 560/590 $\mathrm{nm}(\mathrm{nm})$. The relative fluores cence units were used to generate the quantitative results. All compounds were analyzed as duplicates, and the parameters to calculate $\mathrm{IC}_{50}$ were obtained by GraphPad Prism, version 5.0. $\mathrm{IC}_{50}$ values were determined by the Hill equation rearranged [14].

$\operatorname{LogIC}_{50}=\operatorname{logEC} \mathrm{C}_{50}-\{\log ($ Top-Bottom/Y-Bottom-1)/HillSlope $\}$

\section{Cytotoxicity assay}

The cytotoxicity was measured in L929 and THP1 cell lines using PrestoBlue ${ }^{\circ}$ Cell Viability Reagent (Thermo Scientific, Invitrogen) as described by Lall et al. with modifications [15]. First, cells were maintained in RPMI 1640 (Gibco) supplemented with 10\% fetal bovine serum (FBS) and $1 \%$ antibiotics $(100 \mathrm{U} / \mathrm{mL}$ penicillin, $100 \mu \mathrm{g} / \mathrm{mL}$ streptomycin). Cell cultures were grown and maintained at $37{ }^{\circ} \mathrm{C}$ in a humidified incubator with $5 \% \mathrm{CO}_{2}$. Then, $\mathrm{L} 929$ cells were seeded in 96-wells flat microtiter plate at a concentration of $1 \times 10^{5}$ cells $/ \mathrm{mL}$ (100 $\mu \mathrm{L} /$ well). To THP-1 cells, cultures were differentiated into macrophages by adding $50 \mathrm{ng} / \mathrm{mL}$ 12-myristate 13-acetate phorbol (PMA) (Calbiochem, San Diego, USA) in RPMI-free FBS. After $24 \mathrm{~h}$ of culturing, the adhered cells were washed with PBS and RPMI with 10\% FBS were added. Both cell lines were treated with 4-phenyl-2-aminothiazoles at concentrations ranging from 3.2 to $142 \mu \mathrm{M}$. The plates were incubated for $48 \mathrm{~h}$ and, thereafter, $20 \mu \mathrm{L} /$ well PrestoBlue ${ }^{\circ}$ was added. The plates were incubated for further $2 \mathrm{~h}$ and the fluorescence was read at bottom using $540-570 \mathrm{~nm}$ excitation and 580-610 nm emission. The average of the fluorescence values of the control wells (medium plus PrestoBlue ${ }^{\circ}$ ) was subtracted from the fluorescence value of the positive controls (cells, medium, and PrestoBlue ${ }^{\circ}$ ) and from each experimental well (cells, medium, compounds and PrestoBlue $)^{\circ}$.

Cytotoxicity was also evaluated using Vero cells, a monkey kidney cell line obtained from the American Type Culture Collection (ATCC CCL-81; Manassas, VA). The cytotoxicity was determined by the $3-(4,5 \mathrm{di}-$ methylthiazol- 2-yl)-2,5-diphenyltetrazolium bromide (MTT) method according to a previously described procedure $[16,17]$.

In all cases, the $\mathrm{CC}_{50}$ was calculated as described as in the Antileishmanial assay Section.

\section{Selectivity index calculation}

Selectivity indexes (SI) were calculated by dividing $\mathrm{CC}_{50}$ against each mammalian cell line by the $\mathrm{IC}_{50}$ against $L$. amazonensis $\left(\mathrm{CC}_{50} / \mathrm{IC}_{50}\right)$.

\section{Structure-activity relationship studies}

Regarding the studies of the structure-activity relationship, both the partition coefficient and the molar refractivity (CLogP and MR, respectively) were calculated with Marvin Sketch 6.2.2 software [18]. Electronic parameters, such as punctual Mulliken charges and dipole moment values, were calculated to the minimum energy conformer obtained after geometry optimization processing at Gaussian $09 \mathrm{~W}$ software. Electrostatic potential charges (ChelpG) were calculated by the ab initio method HF/6-31 g* (Gaussian $09 \mathrm{~W}$ ) and mapped on the Connolly surface of each molecule [19]. PaDel-descriptor calculator was used to obtain the topological indexes [20]. Only qualitative structure-activity relationship studies were performed, as well as only the linear correlation coefficients above 0.8 were taken into consideration.

\section{Target fishing and docking studies}

Target fishing studies were performed applying the Web-based Software Pharmmapper [21], considering the 3D-optimized structure of the 4-phenyl-1,3-thiazole-2-amines and the ChelpG charges as the input files. The best 300 results were analyzed based on their fit and z'score functions.

The docking protocol was established after performing redocking studies with the crystallographic data retrieved from Protein Data Bank, entry code 1CG6 (resolution: $1.7 \AA$ ), using the Gold 5.4.1 software [22]. This protocol was applied to the docking simulation with the test compounds, mainly to the 6 one, the best compound of the series. The binding site was defined by the centroid between Met196 and Asp220 and considering a region of $14 \AA$ around this point. Ten runs with 10 solutions each were performed to generate a set of 100 complexes, which were examined by the incidence of a recurrent pose as well as by their Goldscore and Chemscore values.

\section{Results \\ Synthesis}

The compounds were synthesized and isolated as solids of good purity and yields. Structural characterization of these compounds was performed by ${ }^{1} \mathrm{H}-/{ }^{13} \mathrm{C}$-Nuclear Magnetic Resonance (NMR) and Infrared spectra (IR). Gas Chromatography-Mass Spectrometry (GC-MS) analysis was performed to verify the purity, as well as, the structure of the analogues. All compounds presented purities varying from 98 to $100 \%$ and molecular ion values 
$(\mathrm{m} / \mathrm{z})$ and fragmentation patterns correspondent to what was expected for each molecule (Additional file 1).

Compound 1 was obtained as a pale yellow powder (61\%): ${ }^{1} \mathrm{H}-\mathrm{NMR}$ (300 MHz, [D6] DMSO, ppm): $\delta=7.00$ $\left(\mathrm{s}, 1 \mathrm{H}, \mathrm{H}_{5}\right) ; 7.05\left(\mathrm{~s}, 2 \mathrm{H}, \mathrm{NH}_{2}\right) ; 7.24\left(\mathrm{t}, 1 \mathrm{H}, \mathrm{J}_{1}=6 \mathrm{~Hz}, \mathrm{~J}_{2}=\right.$ $\left.9 \mathrm{~Hz}, \mathrm{H}_{13}\right) ; 7.35\left(\mathrm{t}, 2 \mathrm{H}, \mathrm{J}_{1}=6 \mathrm{~Hz}, \mathrm{~J}_{2}=9 \mathrm{~Hz}, \mathrm{H}_{9}\right.$ and $\left.\mathrm{H}_{11}\right)$; $7.79\left(\mathrm{~d}, 2 \mathrm{H}, \mathrm{J}=9 \mathrm{~Hz}, \mathrm{H}_{8}\right.$ and $\left.\mathrm{H}_{12}\right) ;{ }^{13} \mathrm{C}-\mathrm{NMR}(75 \mathrm{MHz}$, [D6] DMSO, ppm): $\delta=101.94\left(\mathrm{C}_{5}\right) ; 125.99\left(\mathrm{C}_{8}\right.$, and $\left.\mathrm{C}_{12}\right)$; $127.62\left(\mathrm{C}_{9}, \mathrm{C}_{10}\right.$ and $\left.\mathrm{C}_{11}\right) ; 128.91\left(\mathrm{C}_{4}\right) ; 135.40\left(\mathrm{C}_{2}\right)$; IR $(\mathrm{KBr}): 3436\left(v_{\text {ass }} \mathrm{NH}_{2}\right) ; 3254\left(v_{\text {sim }} \mathrm{NH}_{2}\right) ; 3155(v \mathrm{C}-\mathrm{H}$ aromatic); $1599(\mathrm{vC}=\mathrm{N}), 715(\mathrm{vC}-\mathrm{S}-\mathrm{C}) ; \mathrm{GC}$ (retention time, relative intensity in \%): 14.10 (100); LRMS (EI, $70 \mathrm{eV}$ ) $m / z$ calculated for $\mathrm{C}_{9} \mathrm{H}_{8} \mathrm{~N}_{2} \mathrm{~S}$ 176.23, found 176 .

Compound 2 was obtained as a pale yellow powder (72\%): ${ }^{1} \mathrm{H}-\mathrm{NMR}$ (300 MHz, [D6] DMSO, ppm): $\delta=2.29$ (s, 3H, $\left.\mathrm{CH}_{3}\right) ; 6.91\left(\mathrm{~s}, 1 \mathrm{H}, \mathrm{H}_{5}\right) ; 7.01\left(\mathrm{~s}, 2 \mathrm{H}, \mathrm{NH}_{2}\right) ; 7.16$ (d, $2 \mathrm{H}, \mathrm{J}=6 \mathrm{~Hz}, \mathrm{H}_{9}$ a and $\left.\mathrm{H}_{11}\right) ; 7.67\left(\mathrm{~d}, 2 \mathrm{H}, \mathrm{J}=9 \mathrm{~Hz}, \mathrm{H}_{8}\right.$ and $\mathrm{H}_{12}$ ); ${ }^{13} \mathrm{C}-\mathrm{NMR}$ (75 MHz, [D6] DMSO, ppm): $\delta=21.25$ $\left(C_{13}\right) ; 101.01\left(C_{5}\right) ; 125.93\left(C_{7}\right) ; 129.48\left(C_{8}\right.$ and $\left.C_{12}\right)$; $132.76\left(\mathrm{C}_{9}\right.$ and $\left.\mathrm{C}_{11}\right) ; 136.81\left(\mathrm{C}_{10}\right) ; 150.36\left(\mathrm{C}_{4}\right) ; 168.53$ $\left(\mathrm{C}_{2}\right)$; IR (KBr): $3454\left(v_{\text {ass }} \mathrm{NH}_{2}\right) ; 3300\left(v_{\text {sim }} \mathrm{NH}_{2}\right) ; 3117$ (vC-H aromatic); $2954\left(v \mathrm{C}-\mathrm{H} \mathrm{CH} \mathrm{CH}_{3}\right) ; 1537(v \mathrm{C}=\mathrm{N}), 731$ (vC-S-C); GC (retention time, relative intensity in \%): 15,46 (100); LRMS (EI, $70 \mathrm{eV}$ ) $\mathrm{m} / z$ calculated for $\mathrm{C}_{10} \mathrm{H}_{10} \mathrm{~N}_{2} \mathrm{~S}$ 190.26, found 190 .

Compound 3 was obtained as a dark yellow powder (59\%): ${ }^{1} \mathrm{H}-\mathrm{NMR}$ (300 MHz, [D6] DMSO, ppm): $\delta=1.18$ $\left(\mathrm{t}, 3 \mathrm{H}, \mathrm{J}_{1}=9 \mathrm{~Hz}, \mathrm{~J}_{2}=6 \mathrm{~Hz}, \mathrm{CH}_{3}\right.$ ethyl); 2.59 (q, $2 \mathrm{H}, \mathrm{J}_{1}=$ $9 \mathrm{~Hz}, \mathrm{~J}_{2}=6 \mathrm{~Hz}, \mathrm{CH}_{2}$ ethyl); $6.91\left(\mathrm{~s}, 1 \mathrm{H}, \mathrm{H}_{5}\right) ; 7.01(\mathrm{~s}, 2 \mathrm{H}$, $\mathrm{NH}_{2}$ ); 7.19 (d, $2 \mathrm{H}, \mathrm{J}=9 \mathrm{~Hz}, \mathrm{H}_{9}$ and $\left.\mathrm{H}_{11}\right) ; 7.69$ (d, $2 \mathrm{H}, \mathrm{J}$ $=9 \mathrm{~Hz}, \mathrm{H}_{8}$ and $\left.\mathrm{H}_{12}\right) ;{ }^{13} \mathrm{C}-\mathrm{NMR}(75 \mathrm{MHz}$, [D6] DMSO, ppm): $\delta=15.97\left(\mathrm{C}_{14}\right) ; 28.35\left(\mathrm{C}_{13}\right) ; 101.06\left(\mathrm{C}_{5}\right) ; 126.00$ $\left(\mathrm{C}_{7}\right) ; 128.27\left(\mathrm{C}_{9}\right.$ and $\left.\mathrm{C}_{11}\right) ; 133.00\left(\mathrm{C}_{8}\right.$ and $\left.\mathrm{C}_{12}\right) ; 143.17$ $\left(\mathrm{C}_{10}\right)$; $150.36\left(\mathrm{C}_{4}\right)$; $168.54\left(\mathrm{C}_{2}\right)$; IR $(\mathrm{KBr}): 3425\left(\mathrm{v}_{\mathrm{ass}} \mathrm{NH}_{2}\right)$; $3265\left(v_{\text {sim }} \mathrm{NH}_{2}\right) ; 3116$ (vC-H aromatic); $2958(v \mathrm{C}-\mathrm{H} \mathrm{CH})_{3}$; $2870\left(v \mathrm{C}-\mathrm{H} \mathrm{CH} \mathrm{CH}_{2}\right) ; 1516(\mathrm{vC}=\mathrm{N})$; GC (retention time, relative intensity in \%): 16,54 (100); LRMS (EI, $70 \mathrm{eV}$ ) MS $m / z$ calculated for $\mathrm{C}_{11} \mathrm{H}_{12} \mathrm{~N}_{2} \mathrm{~S} 204.29$, found 204 .

Compound 4 was obtained as a white powder (53\%): ${ }^{1} \mathrm{H}-\mathrm{NMR}$ (300 MHz, [D6] DMSO, ppm): $\delta=7.14$ (s, 2H, $\left.\mathrm{NH}_{2}\right) ; 7.22\left(\mathrm{~s}, 1 \mathrm{H}, \mathrm{H}_{5}\right) ; 7.61\left(\mathrm{~d}, 1 \mathrm{H}, \mathrm{J}=9 \mathrm{~Hz}, \mathrm{H}_{12}\right) ; 7.77$ (d, $\left.1 \mathrm{H}, \mathrm{J}=9 \mathrm{~Hz}, \mathrm{H}_{11}\right) ; 8.01\left(\mathrm{~d}, 1 \mathrm{H}, \mathrm{J}=3 \mathrm{~Hz}, \mathrm{H}_{8}\right)$; ${ }^{13} \mathrm{C}-\mathrm{NMR}(75 \mathrm{MHz},[\mathrm{D} 6] \mathrm{DMSO}, \mathrm{ppm}): \delta=104.27\left(\mathrm{C}_{5}\right)$; $126.00\left(\mathrm{C}_{12}\right) ; 127.60\left(\mathrm{C}_{7}\right) ; 129.72\left(\mathrm{C}_{8}\right) ; 131.17\left(\mathrm{C}_{11}\right)$; 131.74 ( $\mathrm{C}_{9}$ and $\left.\mathrm{C}_{10}\right)$; $135.88\left(\mathrm{C}_{4}\right)$; $147.65\left(\mathrm{C}_{2}\right)$; IR $(\mathrm{KBr})$ : $3446\left(v_{\text {ass }} \mathrm{NH}_{2}\right) ; 3282\left(v_{\text {sim }} \mathrm{NH}_{2}\right) ; 3124$ ( $\mathrm{vC}-\mathrm{H}$ aromatic); $1527(\mathrm{vC}=\mathrm{N}) ; 1022$ and $1041(\mathrm{vC}-\mathrm{Cl}) ; \mathrm{GC}$ (retention time, relative intensity in \%): 18,96 (97,8); LRMS (EI, $70 \mathrm{eV}$ ) MS $m / z$ calculated for $\mathrm{C}_{9} \mathrm{H}_{6} \mathrm{Cl}_{2} \mathrm{~N}_{2} \mathrm{~S} 245.13$, found 244 .

Compound 5 was obtained as a pale yellow powder (18\%): ${ }^{1} \mathrm{H}-\mathrm{NMR}$ (300 MHz, [D6] DMSO, ppm): $\delta=7.18$ $\left(\mathrm{s}, 1 \mathrm{H}, \mathrm{NH}_{2}\right) ; 7.26\left(\mathrm{~S}, 1 \mathrm{H}, \mathrm{H}_{5}\right) ; 7.73\left(\mathrm{~d}, 2 \mathrm{H}, \mathrm{J}=9 \mathrm{~Hz}, \mathrm{H}_{8}\right.$ and $\left.\mathrm{H}_{12}\right) ; 8.00\left(\mathrm{~d}, 2 \mathrm{H}, \mathrm{J}=9 \mathrm{~Hz}, \mathrm{H}_{9}\right.$ and $\left.\mathrm{H}_{11}\right) ;{ }^{13} \mathrm{C}-\mathrm{NMR}$ (75 MHz, [D6] DMSO, ppm): $\delta=104.84\left(C_{5}\right) ; 125.89$
$\left(\mathrm{C}_{9}\right.$ and $\left.\mathrm{C}_{11}\right) ; 126.46\left(\mathrm{C}_{8}\right.$ and $\left.\mathrm{C}_{12}\right) ; 127.45\left(\mathrm{C}_{10}\right) ; 127.98$ $\left(\mathrm{C}_{7}\right) ; 138.95\left(\mathrm{C}_{13}\right) ; 151.47\left(\mathrm{C}_{4}\right) ; 168.96\left(\mathrm{C}_{2}\right)$; IR $(\mathrm{KBr})$ : $3479\left(v_{\text {ass }} \mathrm{NH}_{2}\right) ; 3298\left(v_{\text {sim }} \mathrm{NH}_{2}\right) ; 3143(v \mathrm{C}-\mathrm{H}$ aromatic); $1537(v \mathrm{C}=\mathrm{N})$; $754(\mathrm{vC}-\mathrm{F}) ; \mathrm{GC}$ (retention time, relative intensity in \%): 14,13 (100); LRMS (EI, $70 \mathrm{eV}$ ) $\mathrm{MS} \mathrm{m} / z$ calculated for $\mathrm{C}_{10} \mathrm{H}_{7} \mathrm{~F}_{3} \mathrm{~N}_{2} \mathrm{~S} 244.24$, found 244 .

Compound 6 was obtained as a pale yellow powder (27\%): mp: 227-230 ${ }^{\circ} \mathrm{C}$; ${ }^{1} \mathrm{H}-\mathrm{NMR}(300 \mathrm{MHz}$, [D6] DMSO, ppm): $\delta=1.29$ (s, 9H, $\mathrm{H}_{14}, \mathrm{H}_{15}$ and $\mathrm{H}_{16}$ ); 6.91 (s, $\left.1 \mathrm{H}, \mathrm{H}_{5}\right) ; 7.02\left(\mathrm{~s}, 2 \mathrm{H}, \mathrm{NH}_{2}\right) ; 7.37\left(\mathrm{~d}, 2 \mathrm{H}, \mathrm{J}=9 \mathrm{~Hz}, \mathrm{H}_{8}\right.$ and $\mathrm{H}_{12}$ ); $7.70\left(\mathrm{~d}, 2 \mathrm{H}, \mathrm{J}=9 \mathrm{~Hz}, \mathrm{H}_{9}\right.$ and $\mathrm{H}_{11}$ ). Melting point: Experimental (227-230 $\left.{ }^{\circ} \mathrm{C}\right)$; Reference $\left(228-230{ }^{\circ} \mathrm{C}\right)$. ${ }^{13} \mathrm{C}-\mathrm{NMR}$, IR and GC-MS acquisitions not performed.

Compound 7 was obtained as a pale yellow powder (47\%): ${ }^{1} \mathrm{H}-\mathrm{NMR}$ (300 MHz, [D6] DMSO, ppm): $\delta=6.74-$ $6.69\left(\mathrm{~m}, 3 \mathrm{H}, \mathrm{H}_{5}, \mathrm{H}_{8}\right.$ and $\left.\mathrm{H}_{12}\right) ; 6.92\left(\mathrm{~s}, 2 \mathrm{H}, \mathrm{NH}_{2}\right) ; 7.60-$ $7.55\left(\mathrm{~m}, 2 \mathrm{H}, \mathrm{H}_{9}\right.$ and $\left.\mathrm{H}_{11}\right) ; 9.42\left(\mathrm{~s}, 1 \mathrm{H}, \mathrm{H}_{13}\right) ;{ }^{13} \mathrm{C}-\mathrm{NMR}$ (75 MHz, [D6] DMSO, ppm): $\delta=98.87\left(\mathrm{C}_{5}\right) ; 115.60\left(\mathrm{C}_{9}\right.$ and $\left.\mathrm{C}_{11}\right) ; 127.35\left(\mathrm{C}_{7}\right) ; 150.57\left(\mathrm{C}_{8}\right.$ and $\left.\mathrm{C}_{12}\right) ; 157.19\left(\mathrm{C}_{10}\right)$; $168.41\left(\mathrm{C}_{4}\right) ; 206.96\left(\mathrm{C}_{2}\right)$; IR $(\mathrm{KBr})$ : $3487\left(v_{\text {ass }} \mathrm{NH}_{2}\right) ; 3377$ $\left(v_{\text {sim }} \mathrm{NH}_{2}\right) ; 3126$ (vC-H aromatic); 2984 (vO-H); 1535 $(v \mathrm{C}=\mathrm{N})$; $\mathrm{GC}$ (retention time, relative intensity in \%): 17,69 (100); LRMS (EI, $70 \mathrm{eV}$ ) $\mathrm{MS} \mathrm{m} / z$ calculated for $\mathrm{C}_{9} \mathrm{H}_{8} \mathrm{~N}_{2} \mathrm{OS}$ 192.24, found 192 .

Compound 8 was obtained as a white powder (67\%): ${ }^{1} \mathrm{H}-\mathrm{NMR}$ (300 MHz, [D6] DMSO, ppm): $\delta=7.07$ (s, 1H, $\left.\mathrm{H}_{5}\right) ; 7.08\left(\mathrm{~s}, 2 \mathrm{H}, \mathrm{NH}_{2}\right) ; 7.39-7.44\left(\mathrm{~m}, 2 \mathrm{H}, \mathrm{H}_{8}\right.$ and $\left.\mathrm{H}_{9}\right)$; 7.83-7.79 (m, 2H, $\mathrm{H}_{9}$ and $\left.\mathrm{H}_{11}\right) ;{ }^{13} \mathrm{C}-\mathrm{NMR}(75 \mathrm{MHz}$, [D6] DMSO, ppm): $\delta=102.77\left(\mathrm{C}_{5}\right) ; 127.69\left(\mathrm{C}_{7}\right) ; 128.93$ $\left(\mathrm{C}_{9}\right.$ and $\left.\mathrm{C}_{11}\right) ; 131.98\left(\mathrm{C}_{8}\right.$ and $\left.\mathrm{C}_{12}\right) ; 134.23\left(\mathrm{C}_{10}\right) ; 143.05$ $\left(\mathrm{C}_{4}\right) ; 168.81 \quad\left(\mathrm{C}_{2}\right)$; IR $(\mathrm{KBr}): 3437 \quad\left(v_{\text {ass }} \mathrm{NH}_{2}\right) ; 3282$ $\left(v_{\text {sim }} \mathrm{NH}_{2}\right) ; 3109$ ( $v \mathrm{C}-\mathrm{H}$ aromatic); $1533(v \mathrm{C}=\mathrm{N}) ; 826$ ( $v \mathrm{C}-\mathrm{Cl})$; $\mathrm{GC}$ (retention time, relative intensity in \%): 16,50 (99,6); LRMS (EI, $70 \mathrm{eV}$ ) MS $\mathrm{m} / z$ calculated for $\mathrm{C}_{9} \mathrm{H}_{7} \mathrm{ClN}_{2} \mathrm{~S} 210.68$, found 210 .

\section{Biological assays}

All compounds were tested against L. amazonensis promastigotes. The cytotoxicity was also assessed against L929 and THP-1 by using the PrestoBlue ${ }^{\oplus}$ cell viability reagent, as well as against Vero cell lines by using the MTT viability assay. The results are presented in Table 2.

\section{Structure-activity relationships and molecular modeling studies}

Structure-activity relationship analyses revealed a good parabolic correlation between CLogP and antileishmanial activity (Additional file $2 \mathrm{~A}$ ), with a correlation coefficient of 0.968 . The same behavior was also observed when regarding the Molar Refractivity (MR) values of the compounds, a parameter which also describes molecular hydrophobicity associated to molecular volume (Additional file 2B). 
Table $2 \mathrm{IC}_{50}$ values against L. amazonensis promastigotes and the cytotoxicity assay results, expressed as $C_{50}$ values

\begin{tabular}{|c|c|c|c|c|c|c|c|c|}
\hline Compound & $I_{50}(\mu \mathrm{M})$ & $\mathrm{plC}_{50}$ & $\mathrm{CC}_{50}(\mu \mathrm{M}) \mathrm{THP} 1$ cells $^{\mathrm{a})}$ & $\mathrm{SI}$ & $\mathrm{CC}_{50}(\mu \mathrm{M})$ L929 cells $^{\mathrm{b})}$ & $\mathrm{SI}$ & $\mathrm{CC}_{50}(\mu \mathrm{M}) \mathrm{VERO}$ cells $^{\mathrm{c})}$ & $\mathrm{SI}$ \\
\hline 1 & 957.56 & 3.02 & 143.57 & 0.15 & 198.26 & 0.20 & 710.06 & 0.74 \\
\hline 2 & 107.68 & 3.97 & 135.40 & 1.26 & 159.52 & 1.48 & 657.51 & 6.11 \\
\hline 3 & 46.63 & 4.33 & 117.27 & 2.51 & 95.45 & 2.05 & 1217.5 & 26.11 \\
\hline 4 & 53.12 & 4.27 & 84.65 & 1.59 & 121.12 & 2.28 & 255.03 & 4.80 \\
\hline 5 & 53.37 & 4.27 & 92.21 & 1.73 & 106.08 & 2.00 & 511.76 & 9.59 \\
\hline 6 & 20.78 & 4.68 & 45.73 & 2.20 & 27.07 & 1.30 & 118.17 & 5.69 \\
\hline 7 & inactive & inactive & inactive & ND & Inactive & ND & 651.29 & ND \\
\hline 8 & 115.95 & 3.94 & 82.15 & 0.70 & 115.06 & 0.99 & 595.94 & 5.14 \\
\hline Amphotericin B & 16.23 & - & ND & - & ND & - & ND & - \\
\hline Pentamidine & 10.76 & - & ND & - & ND & - & ND & - \\
\hline
\end{tabular}

(a) THP1 cells: human monocytic cell line derived from acute monocytic leukemia patients; (b) L929 cells: fibroblasts from subcutaneous connective tissue; c) Vero cells: kidney epithelial cells extracted from African green monkeys. $I C_{50}$ : Half-maximal inhibitory concentration on promastigotes; pIC $C_{50}$ : Half-maximal inhibitory concentration in log units; $\mathrm{CC}_{50}$ : Half cytotoxic concentration; SI: Selectivity Indexes, ND: Not determined. pIC 50 calculates as pIC $\mathrm{C}_{50}=-\mathrm{log}_{10}\left(\mathrm{IC}_{50}\right)$

No significant correlation $\left(\mathrm{r}^{2}=>0.8\right)$ was found between activity and electronic parameters, such as electrostatic potential charges, punctual charges or dipole moments (see Additional file 3 to verify the parameters and Additional file 4 for electrostatic potential maps).

However, some relationships could be observed regarding the Electrostatic Potential Maps. Compounds $\mathbf{3}$ and $\mathbf{6}$, for instance, showed a green left-side region (Additional file 4) whereas compounds $\mathbf{4}$ and $\mathbf{5}$, which presented an intermediary activity, exhibited a yellowish left-side region similarly to the one observed with compound 7 , an inactive analogue. These findings could reveal some potential influence of the electronic distribution on the activity, although further studies must be performed to confirm these relationships.

As the cytotoxic effects on Vero cells were substantially different from those obtained against $L$. amazonensis, L-929 and THP-1 cells, a target fishing study was proposed to suggest potential targets and, consequently, mechanisms which could explain these observations.

The web-based software Pharmmapper was applied to search pharmacophore databases, such as the Target Bank, Drug Bank, Binding DB, and Potential Drug Target Database. Such software also takes advantage of a normalized score function, the z'score, to present the most probable targets to compounds with defined chemical structures [21].

The energetically optimized structure of each compound, considering their ChelpG charges, was employed as input, and the best 300 targets - human and non-human - were retrieved. Table 3 lists the five best targets common to these compounds, along with the maximum z'score values that were obtained.

The human S-Methyl-5-thioadenosine phosphorylase was elected as the best potential target, based in two criteria: the best Max z'score values found to each compound under analysis combined to the fact that this target was returned to all compound submitted to the target fishing.

A BLAST (Basic Local Alignment Search Tool, National Center for Biotechnology Information) search was, then, performed to find homologous proteins in the L. amazonensis available proteome [23]. The best nine results are listed in Table 4, together with the statistical analyses which corroborate their significance.

Identity values above $35 \%$ were observed for the Leishmania species enzymes, so a docking study considering the human enzyme was also performed to elucidate the potential binding mode of the compounds into the catalytic site. The results are illustrated in Fig. 1.

Table 3 Pharmmapper results

\begin{tabular}{|c|c|c|c|c|}
\hline Target & PDB Code & Ligand Compounds & Max z'score value ${ }^{a}$ & Function \\
\hline S-Methyl-5-thioadenosine phosphorylase & $1 C G 6$ & All derivatives & 3.69 & Nucleotide transport and metabolism \\
\hline Adenosine deaminase & $1 \mathrm{~V} 79 / 1$ V89 & All but compound $\mathbf{3}$ & 4.72 & Nucleotide transport and metabolism \\
\hline UvrABC System Protein B & $1 C 40$ & All but compound 4 & 3.59 & Replication, recombination, and repair \\
\hline Dihydrofolate reductase & $1 \mathrm{~A} 2$ & All but compound 4 & 3.12 & Coenzyme transport and metabolism \\
\hline Queuine t-RNA-ribosyltransferase & 1Q66 & $\begin{array}{l}\text { All but compounds } \mathbf{3} \\
\text { and } \mathbf{4}\end{array}$ & 3.02 & $\begin{array}{l}\text { Translation, ribosomal structure and } \\
\text { biogenesis }\end{array}$ \\
\hline
\end{tabular}

(a) among all compounds tested 
Table 4 BLAST results for Leishmania sp protein sequences producing significant alignment with human S-methyl-5-thioadenosine phosphorylase

\begin{tabular}{|c|c|c|c|c|c|}
\hline Target & Max Score & Total Score & Query Cover (\%) & E value & Identity (\%) \\
\hline Putative methylthioadenosine phosphorylase [Leishmania infantum JPCM 5] & 182 & 182 & 87 & $2 e-55$ & 38 \\
\hline Putative methylthioadenosine phosphorylase [L. major strain Friedlin] & 180 & 180 & 87 & $1 e-54$ & 38 \\
\hline Putative methylthioadenosine phosphorylase [L. guyanensis] & 182 & 182 & 95 & $3 e-55$ & 37 \\
\hline Putative methylthioadenosine phosphorylase [L. mexicanaMHOM/GT/2001/U1103] & 182 & 182 & 95 & $2 e-55$ & 37 \\
\hline Putative methylthioadenosine phosphorylase [L. panamensis] & 179 & 179 & 95 & $2 e-54$ & 37 \\
\hline Putative methylthioadenosine phosphorylase [L. brasiliensis MHOM/BR/75/M2904] & 179 & 179 & 95 & $2 e-54$ & 37 \\
\hline
\end{tabular}

\section{Discussions}

The compounds were obtained as expected with good yields and purities. The fact of the easy synthetic preparation constitutes one of the major advantages for the future application of these analogues as biological agents, mainly regarding the therapeutics of neglected diseases.

Out of the eight tested analogues, four of them showed activity against the promastigotes of L. amazonensis (Table 2), within the range of 20 to $60 \mu \mathrm{M}$. Compound 6 was the most active one $(21 \mu \mathrm{M})$, exhibiting an $\mathrm{IC}_{50}$ value close to the one obtained with the standard drug of the assay, the Amphotericin B $(16.23 \mu \mathrm{M})$. Compound 3 was the second best of the series, followed by Compounds $\mathbf{4}$ and $\mathbf{5}$, both presenting very similar antileishmanial behavior. However, no compound was better than the pentamidine standard drug $(10.76 \mu \mathrm{M})$.

Even though compounds that were more active against leishmaniasis have been reported in literature, our results are still encouraging because the antileishmanial activity was verified solely considering this scaffold.

A suitable cytotoxic profile was obtained to compound 6 when considering the THP-1 cells, with a selectivity index of 2.20, but this index was lower when considering L929 cells (1.30). The THP-1 cell line is a frequent model for imitating the function and regulation of macrophages, the host cells of $L$. amazonensis.

Similarly, Compounds 3, 4 and 5 exhibited selectivity indexes suitable to proceed to the next steps of the development, since the toxic dose was about two times higher than the effective dose, both when considering L929 and THP-1 cells. Moreover, no cytotoxic effect was observed against Vero cells at the same concentration range tested, except for compound 1, which had a SI of 0.74 . This last compound was inactive against L. amazonensis, though.

These observations encouraged us to proceed with further studies, aiming to understand which of the
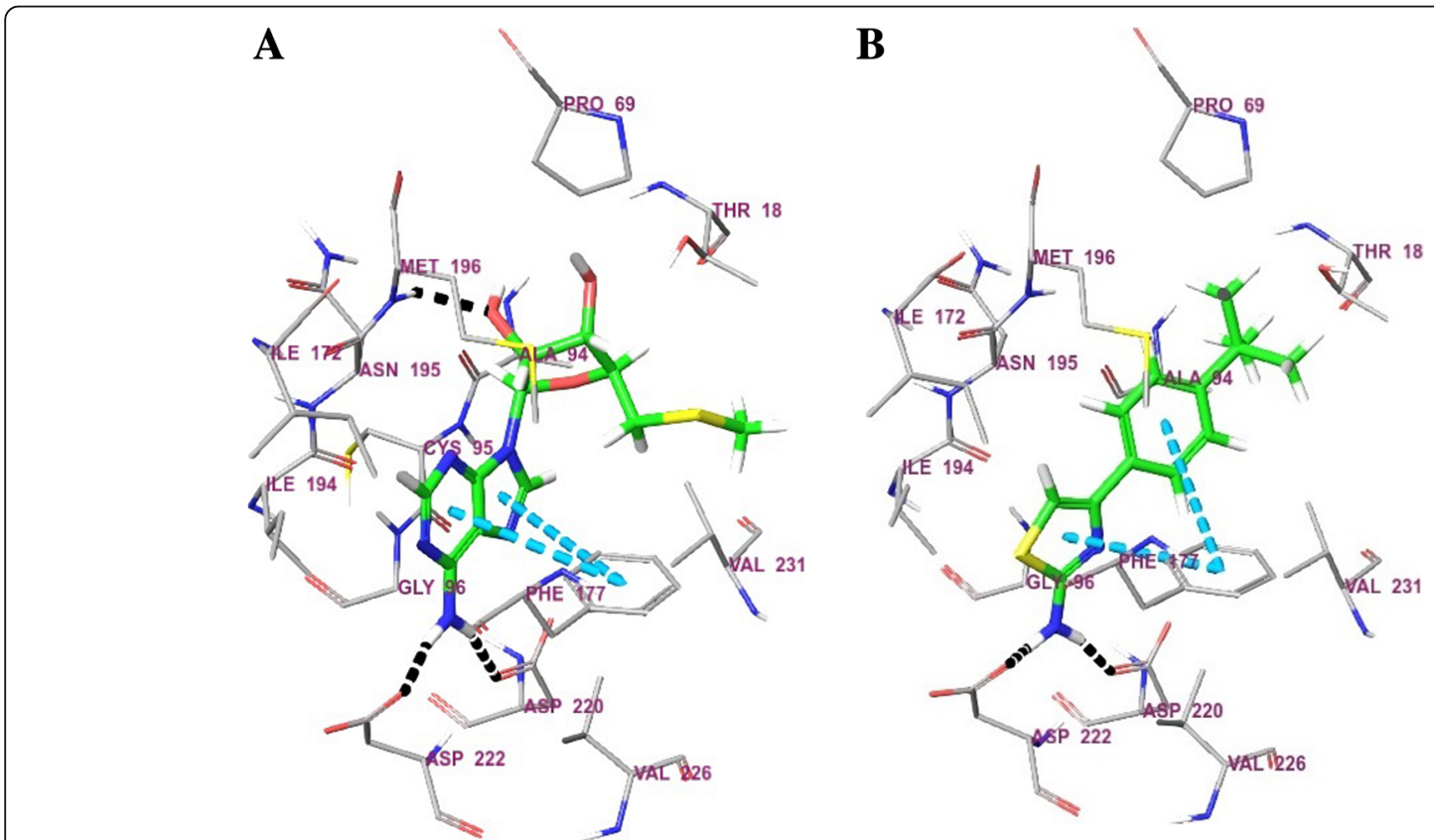

Fig. 1 Docking at the S-methyl-5-thioadenosine phosphorylase. (a) Co-crystallized ligand. (b) Compound $\mathbf{6}$ docked at the active site. Blue dashed lines: pi-stacking interactions; black dashed lines: hydrogen bonds (For color version, please access the online version of the article) 
structural aspects could be related to the activity or cytotoxicity of the compounds.

CLogP values ranging from 3.5 to 4.5 seem to be ideal to the biological activity of the series observed. Moreover, molar refractivity (MR) also presented a good correlation with antileishmanial activity (Additional file 2), indicating that not just the lipophilicity contributes to the antileishmanial activity, but also molar volume and polarizability.

The observed relations with hydrophobic, volume, and polarizability parameters strongly suggest the importance of membrane permeation to the activity of these scaffolds.

Such findings are also in accordance with the observations of Papadopoulou et al., in which linear correlation coefficients of 0.979 and 0.977 were found between the antitrypanosomal activity and the CLogP values of their piperazine and non-piperazine derivatives [10]. Their results against $L$. donovani are significant and have also demonstrated good correlation with the lipophilicity of their analogues $\left(r^{2}=0.886\right)$. These compounds, however, presented cytotoxicity toward L6 cells, which were also related to the lipophilicity.

Aiming to explain the differential cytotoxic profile observed among the eukaryotic cells, molecular modeling studies were also performed.

Initially, a target fishing study was carried out to identify potential targets to the compounds. Most of the returned targets are related to nucleotide recognition, processing, transport, and/or metabolism. This could indicate structural similarity between the scaffold and these endogenous substrates. It also could explain, to some degree, the observed cytotoxicity toward some of the tested cell lines.

The single common target to all tested compounds was the S-methyl-5-thioadenosine phosphorylase (1CG6, pdb entry). This enzyme catalyzes the conversion of 5 '-deoxy-5'-methylthioadenosine in adenine and 5-met hylthio-D-ribose-1-phosphate in a fundamental step of the polyamine biosynthesis [24]. This pathway is essential to the production of nucleotides and, therefore, essential to cell growth and proliferation.

Unfortunately, the phosphorylase isoform pointed out as a target to these compounds is the human one, and it would not be able to explain the antileishmanial action observed. However, this enzyme has its correspondent isoform in several living organisms.

Investigation for potential Leishmania $s p$ isoforms was performed with the BLAST. The search found nine protein sequences with identity values ranging from 37 to $38 \%$, all from different Leishmania species, including one from the L. mexicana complex. The Expectation values (E-values) of $10^{-54}$ obtained in this study corroborates with the significance of the alignment, since the lower the E-value, the more significant the alignment and its scores (Table 4).

Most of the conserved residues are located at the binding site region, as revealed by the alignment of the putative L. mexicana phosphorylase and the human isoform (Fig. 2).

The binding mode of $5^{\prime}$-deoxy-5'-methylthioadenosine on the human isoform is sustained by three main hydrogen-bound interactions with the Met196, Asp220 and Asp222. The alignment with the enzyme from $L$. mexicana showed that these residues are conserved at the binding site, corresponding to the Met200, Asp224 and Asp226.

This similarity could be the basis for the cytotoxicity observed toward the L929 fibroblasts and THP-1 cells, but not toward the Vero cells, which is a S-methyl-5-thioadenosine phosphorylase negative cell line [25].

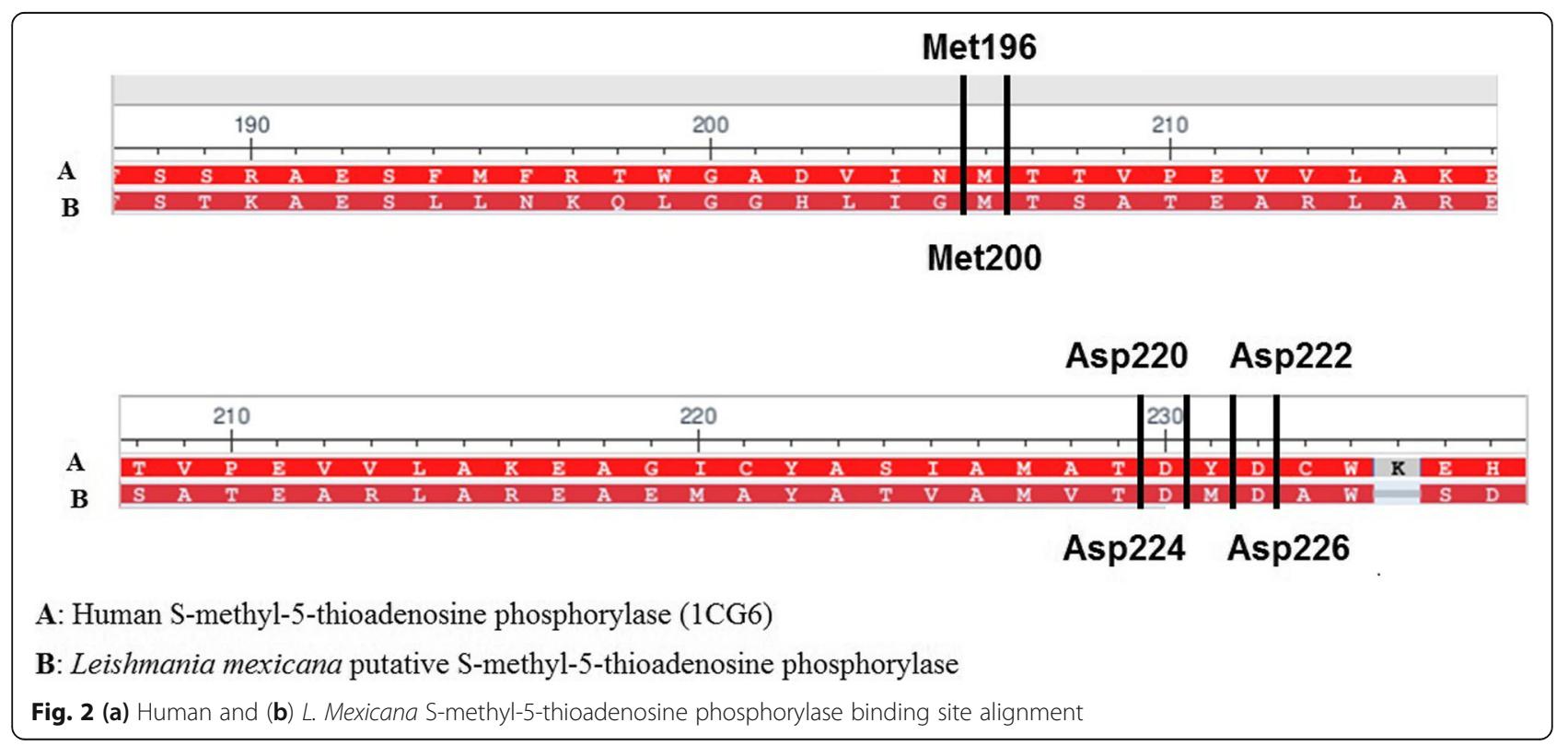


In the absence of crystallographic data from the $L$. mexicana enzyme, docking studies with the human isoform were performed to verify the potential binding mode of the 4-phenyl-2-aminothiazole to the phosphorylase (Fig. 1).

The most prevalent pose of compound $\mathbf{6}$ at the phosphorylase binding site was found at $89 \%$ frequency. This pose showed values 43.34Goldscore and 18.93 Chemscore, which are compatible with the good fitness of compound $\mathbf{6}$ at the binding site.

The docked compound $\mathbf{6}$ also showed good correspondence with the binding mode exhibited by the $5^{\prime}$-deoxy-5'-methylthioadenosine, the original ligand of the phosphorylase.

The amino group of 4-phenyl-2-aminothiazole compound 6 (Fig. 1b) established hydrogen bonds, as a donor, with the Asp220 and Asp222 residues, just like observed for the co-crystallized ligand (Fig. 1a). Compound 6 also positioned its aromatic systems suitably to establish pi-stacking interactions with Phe117, which, once more, mimics the 5 '-deoxy-5' -methylthioadenosine binding mode. The tert-butyl group from the 4-phenyl ring was positioned close to a set of hydrophobic residues, Thr18, Pro69 and Val231, occupying the same site of the methylthio moiety from the co-crystallized ligand. Conversely, no interaction between compound $\mathbf{6}$ and Met196 was observed.

Compounds 3, 4 and 5 were also theoretically docked to the phosphorylase and exhibited the same pattern of interactions described above when regarding their most frequent poses, $96 \%, 94 \%$ and $100 \%$, respectively (Additional file 5).

Literature on S-methyl-5-thioadenosine phosphorylase expression in different cell lines reports that almost all non-neoplastic mammal cells express this enzyme [26-28]. Most of the S-methyl-5-thioadenosine phosphorylase lacking cells are malignant cells, as discussed by Kamatani et al. [29].

Even though these studies were performed taking the human enzyme into consideration, the results could be used as a guide to future design new antileishmanial compounds. For instance, homology modeling studies can be useful in order to improve such studies.

Derivatives of 2-aminothiazoles have been reported as predominantly active against trypanosomes, such as in the case of Kaiser et al., but they have not shown expressive activity against leishmanias. Nearly all literature reports are based on studies with $L$. donovani or $L$. infantum, both responsible for the visceral leishmaniasis, since this is the lethal form of the disease [9].

Unfortunately, cutaneous leishmaniasis is thought to be less dangerous, and because of it, it is believed to potentially lead to a spontaneous cure. Several cases, however, evolve to the mucocutaneous form of the diseases, just like observed in infections caused by the $L$. braziliensis. This clinical manifestation is characterized by deforming lesions that often compromise the patient's social life.

Moreover, leishmania species from the L. mexicana complex are widely known to be resistant to treatment, mainly those based on in situ free-radical production, as is the case of nitroderivatives. Such mechanism of action implies low selectivity and, consequently, a broad panel of side effects. Ironically, several of the promising new compounds explored against trypanosomatidae parasites are nitroaromatic compounds [30,31].

Finding compounds that are active against such parasites with $\mathrm{IC}_{50}$ values lower than $10 \mu \mathrm{M}$ and with good toxicological profile is still a challenging task. From this point of view, the 4-phenyl-2-aminothiazole scaffold arises as a non-nitroaromatic alternative to the development of new antileishmanial drugs.

\section{Conclusion}

Considering the results herein reported, the 4-phenyl -2-aminothiazoles might be potential scaffolds to be explored in the search for new antileishmanial hits. Four out of the eight tested compounds exhibited important anti-promastigote activity associated with good selectivity indexes, except in the cases of compounds $\mathbf{1}$ and $\mathbf{6}$ (in the latter, only when considering its activity against L929 cells).

This is the first report of their effectiveness against the L. amazonensis, one of the species of leishmania responsible for the cutaneous form of the disease. Currently, studies using the most promising compounds have been conducted to evaluate their effects on the amastigote form of the parasite, which is found in the mammalian host. Such tests are important and fundamental for a better evaluation of the applicability of the compounds for the treatment of leishmaniasis.

The 4-phenyl-2-aminothiazole scaffold arises, as discussed, as a non-nitroaromatic alternative to the development of new antileishmanial drugs. As scaffolds, these structures could be rationally modified to present selectivity along with improved antileishmanial activity, since the differences between the binding sites of the human and leishmania phosphorylases can be explored.

\section{Additional files}

Additional file 1: GC-MS results, under the following conditions: Inlet temperature: $250{ }^{\circ} \mathrm{C}$; Oven: initial temperature $80^{\circ} \mathrm{C}$, $10{ }^{\circ} \mathrm{C} / \mathrm{min}$ up to $250^{\circ} \mathrm{C}$, kept for $13 \mathrm{~min}$; Column RTX-5MS (30 $\left.\mathrm{m} \times 0.25 \mathrm{~mm} \times 0.25 \mu \mathrm{m}\right)$. (DOCX 342 kb)

Additional file 2: Structure-Activity Relationships. (a) CLogP versus Biological Activity Graphic. Parabolic Correlation coefficient, $r^{2}=0.968$. (b) MR versus Biological Activity Graphic. Parabolic Correlation coefficient, $r^{2}$ $=0.945$. (DOCX $40 \mathrm{~kb}$ ) 
Additional file 3: Physical chemical parameters calculated. (DOCX $14 \mathrm{~kb}$ )

Additional file 4: Electrostatic potential maps. (DOCX $2496 \mathrm{~kb}$ )

Additional file 5: GPQF-03, GPQF-04, and GPQF-05 best docking poses at S-methyl-5-thioadenosine phosphorylase (1CG6). (DOCX 284 kb)

\section{Acknowledgments}

The authors are grateful to FAPESP for the financial support (FAPESP 2013/ 01875-0), and to CAPES and CNPq for the grants.

\section{Funding}

The authors would like to thank the São Paulo Research Foundation (FAPESP) for the financial support (FAPESP 2013/01875-0) and for the software licenses, when applicable; and the Coordination for the Improvement of Higher Education Personnel (CAPES) and the National Council for Scientific and Technological Development (CNPq) for the grants of MOLC and PFS (PIBIC).

The publication of this work was also partially supported by the Coordination for the Improvement of Higher Education Personnel (CAPES) through Programa Editoração CAPES (edital n. 13/2016, auxílio n. 0722/2017, processo n. 88881.142062/2017-01) and by the National Council for Scientific and Technological Development (CNPq) through Programa Editorial CNPq/ CAPES (chamada n. 26/2017, processo n. 440954/2017-7)

\section{Availability of data and materials}

Not applicable.

\section{Authors' contributions}

The authors contributed equally to the final version of the manuscript. CAR, PFdS, MOLdC, and TFAP developed the synthetic, structural and activity relationships and molecular modeling studies under the supervision of Dr. DGGR. Dr. PX and MMG were in charge of both the antileishmanial assays and the cytotoxicity assay against THP-1 and L929 cell lines. Dr. JdM and AM developed the cytotoxicity assays on Vero cells. All authors read and approved the final manuscript.

\section{Ethics approval}

Not applicable.

\section{Consent for publication}

Not applicable.

\section{Competing interests}

The authors declare that they have no competing interests.

\section{Publisher's Note}

Springer Nature remains neutral with regard to jurisdictional claims in published maps and institutional affiliations.

\section{Author details \\ ${ }^{1}$ Chemical and Pharmaceutical Research Group, Department of Pharmaceutical Sciences, Institute of Environmental, Chemical and Pharmaceutical Sciences, Federal University of São Paulo (UNIFESP), Rua São Nicolau, 210, 20 andar, Diadema, SP 09913-030, Brazil. ' Laboratory of Cellular Immunology and Biochemistry of Fungi, Department of Pharmaceutical Sciences, Institute of Environmental, Chemical and Pharmaceutical Sciences, Federal University of São Paulo (UNIFESP), Rua São Nicolau, 210, 20 andar, Diadema, SP 09913-030, Brazil. ${ }^{3}$ Research Group of Neglected Diseases, University of Guarulhos, Praça Tereza Cristina, 88, Guarulhos, SP 07020-071, Brazil.}

Received: 26 April 2018 Accepted: 28 August 2018

Published online: 10 September 2018

\section{References}

1. World Health Organization - Leishmaniasis. http://www.who.int/ leishmaniasis/en/. Accessed 24 Jan 2016.

2. World Health Organization. Control of the leishmaniasis: report of a meeting of the WHO Expert Committee on the Control of Leishmaniases, Geneva; 2010. p. 55-65.
3. Croft SL. Public-private partnership: from there to here. Trans R Soc Trop Med Hyg. 2005;99(SUPPL 1):S9-14.

4. Ghaemmaghami S, May BCH, Renslo AR, Prusiner SB. Discovery of 2aminothiazoles as potent antiprion compounds. J Virol. 2010;84(7):3408-12. https://doi.org/10.1128/JVl.02145-09.

5. Amnerkar ND, Bhusari KP. Synthesis of some thiazolyl aminobenzothiazole derivatives as potential antibacterial, antifungal and anthelmintic agents. J Enzyme Inhib Med Chem. 2011;26(1):22-8.

6. Khan KM, Ambreen N, Karim A, Saied S, Amyn A, Ahmed A, et al. Schiff bases of thiazole as antibacterial and antifungal agents. J Pharm Res. 2012; 5(1):651-6.

7. Gorczynski MJ, Leal RM, Mooberry SL, Bushweller JH, Brown ML. Synthesis and evaluation of substituted 4-aryloxy- and 4-arylsulfanyl-phenyl-2aminothiazoles as inhibitors of human breast cancer cell proliferation. Bioorg Med Chem. 2004;12(5):1029-36.

8. Karabasanagouda T, Vasudeva A. Synthesis of some new 2-(4alkylthiophenoxy)-4-substituded-1,3-thiazoles as possible anti-inflammatory and antimicrobial agents. Indian J Chem. 2008;47(1):144-52.

9. Kaiser M, Maes L, Tadoori LP, Spangenberg T, loset JR. Repurposing of the open access malaria box for kinetoplastid diseases identifies novel active scaffolds against trypanosomatids. J Biomol Screen. 2015;20(5):634-45.

10. Papadopoulou MV, Bloomer WD, Rosenzweig HS, Wilkinson SR, Szular J, Kaiser M. Antitrypanosomal activity of 5-nitro-2-aminothiazole-based compounds. Eur J Med Chem. 2016;117:179-86. https://doi.org/10.1016/j. ejmech.2016.04.010

11. Bilbao-Ramos P, Galiana-Roselló C, Dea-Ayuela MA, González-Alvarez M, Vega C, Rolón M, et al. Nuclease activity and ultrastructural effects of new sulfonamides with anti-leishmanial and trypanocidal activities. Parasitol Int. 2012;61(4):604-13. https://doi.org/10.1016/j.parint.2012.05.015.

12. Hantzsch A, Weber JH. Ueber Verbindungen des Thiasola (Pyridine der Thiophenreihe). Dtsch Chem Gesellschaft. 1887;20:3118-32.

13. Carroll King L, Hlavacek RJ. The reaction of ketones with iodine and thiourea. J Am Chem Soc. 1950;72(8):3722-5.

14. Directorate E, Meeting J, The OF, Committee C, Working THE, On P. Guidance document on using cytotoxicity tests to estimate starting doses for acute oral systemic toxicity tests. Organ Econ Co-operation Dev [Internet]. 2010;129:1-54. Available from: http://www.oecd.org/ officialdocuments/displaydocumentpdf?cote=env/jm/ mono(2010)46\&doclanguage $=$ en

15. Lall N, Henley-Smith CJ, De Canha MN, Oosthuizen CB, Berrington D. Viability reagent, prestoblue, in comparison with other available reagents, utilized in cytotoxicity and antimicrobial assays. Int J Microbiol. 2013;2013: Article ID 420601

16. de Moraes J, Dario BS, Couto RAA, Pinto PLS, da Costa Ferreira AM. Antischistosomal activity of oxindolimine-metal complexes. Antimicrob Agents Chemother. 2015;59(10):6648-52.

17. de Brito MRM, Peláez WJ, Faillace MS, Militão GCG, Almeida JRGS, Argüello GA, et al. Cyclohexene-fused 1,3-oxazines with selective antibacterial and antiparasitic action and low cytotoxic effects. Toxicol in Vitro. 2017:44:273-9. https://doi.org/10.1016/j.tiv.2017.07.021

18. MarvinSketch (version 6.2.2), calculation module developed by ChemAxon. 2014. http://www.chemaxon.com/products/marvin/marvinsketch/.

19. Frisch MJ, Trucks GW, Schlegel HB, Scuseria GE, Robb MA, Cheeseman JR, et al. Gaussian 09. Revision D.1. Wallingford: Gaussian, Inc; 2009.

20. Yap CW. PaDEL-descriptor: an open source software to calculate molecular descriptors and fingerprints. J Comput Chem. 2011;32(7):1466-74.

21. Liu X, Ouyang S, Yu B, Liu Y, Huang K, Gong J, et al. PharmMapper server: a web server for potential drug target identification using pharmacophore mapping approach. Nucleic Acids Res. 2010;38(Web Server issue):W609-14.

22. Jones $G$, Willett $P$, Glen RC, Leach AR, Taylor R. Development and validation of a genetic algorithm for flexible docking. J Mol Biol. 1997;267(3):727-48. https://doi.org/10.1006/jmbi.1996.0897.

23. Altschul SF, Gish W, Miller W, Myers EW, Lipman DJ. Basic local alignment search tool. J Mol Biol. 1990;215(3):403-10. https://doi.org/10.1016/S00222836(05)80360-2.

24. Appleby TC, Erion MD, Ealick SE. The structure of human 5'-deoxy-5'methylthioadenosine phosphorylase at 1.7 a resolution provides insights into substrate binding and catalysis. Structure. 1999:7(6):629-41.

25. Della Ragione F, Russo G, Oliva A, Mastropietro S, Mancini A, Borrelli A, et al. 5'-Deoxy-5'-methylthioadenosine phosphorylase and p16INK4 deficiency in multiple tumor cell lines. Oncogene. 1995;10(5):827-33. 
26. Williams-Ashman HG, Seidenfeld J, Galletti P. Trends in the biochemical pharmacology of 5'-deoxy-5'-methylthioadenosine. Biochem Pharmacol. 1982;31(3):277-88.

27. Carrera CJ, Eddy RL, Shows TB, Carson DA. Assignment of the gene for methylthioadenosine phosphorylase to human chromosome 9 by mousehuman somatic cell hybridization. Proc Natl Acad Sci U S A. 1984;81(9): 2665-8.

28. Della Ragione F, Oliva A, Palumbo R, Russo GL, Gragnaniello V, Zappia V. Deficiency of 5'-deoxy-5'-methylthioadenosine phosphorylase activity in malignancy. Absence of the protein in human enzyme-deficient cell lines. Biochem J. 1992;281(Pt 2):533-8. https://doi.org/10.1042/bj2810533.

29. Kamatani N, Nelson-Rees WA, Carson DA. Selective killing of human malignant cell lines deficient in methylthioadenosine phosphorylase, a purine metabolic enzyme. Proc Natl Acad Sci U S A. 1981;78(2):1219-23. https://doi.org/10.1073/pnas.78.2.1219.

30. Rando DG, Avery MA, Tekwani BL, Khan SI, Ferreira El. Antileishmanial activity screening of 5-nitro-2-heterocyclic benzylidene hydrazides. Bioorg Med Chem. 2008;16(14):6724-31.

31. Soong L, Henard CA, Melby PC. Immunopathogenesis of non-healing American cutaneous leishmaniasis and progressive visceral leishmaniasis. Semin Immunopathol. 2012;34(6):735-51.

Ready to submit your research? Choose BMC and benefit from:

- fast, convenient online submission

- thorough peer review by experienced researchers in your field

- rapid publication on acceptance

- support for research data, including large and complex data types

- gold Open Access which fosters wider collaboration and increased citations

- maximum visibility for your research: over $100 \mathrm{M}$ website views per year

At $\mathrm{BMC}$, research is always in progress.

Learn more biomedcentral.com/submissions 